\title{
The Golden Hour: a quality improvement initiative for extremely premature infants in the neonatal intensive care unit
}

\author{
Sarah E. W. Croop ${ }^{1,2} \cdot$ Suzanne M. Thoyre ${ }^{2} \cdot$ Sofia Aliaga ${ }^{1} \cdot$ Martin J. McCaffrey $^{1} \cdot$ Sigal Peter-Wohl $^{1}$
}

Received: 18 May 2019 / Revised: 21 October 2019 / Accepted: 28 October 2019 / Published online: 11 November 2019

(c) Springer Nature America, Inc. 2019

\begin{abstract}
Background Following delivery, extremely premature infants are vulnerable to rapid development of hypothermia and hypoglycemia. To reduce local rates of these morbidities, a multidisciplinary team developed a protocol standardizing evidence-based care practices during the first hour after birth.

Methods Using quality improvement methodology, the Golden Hour protocol was implemented for all inborn infants $<27$ weeks' gestation. Data were collected (2012-2017) over three phases; pre-protocol $(n=80)$, Phase I $(n=42)$, and Phase II $(n=92)$.

Results There were no significant differences in infant characteristics. Improvements in hypothermia (59\% vs $26 \%$ vs $38 \%$; $p=0.001)$, hypoglycemia ( $18 \%$ vs $7 \%$ vs $4 \% ; p=0.012)$, and minutes to completion of stabilization [median $(\mathrm{Q} 1, \mathrm{Q} 3) 110$ $(89,138)$ vs $111(94,135)$ vs $92(74,129) ; p=0.0035]$ were observed.

Conclusions Implementation of an evidence-based, Golden Hour protocol is an effective intervention for reducing hypothermia and hypoglycemia in extremely premature infants.
\end{abstract}

\section{Introduction}

Prematurity is the leading cause of neonatal death and contributes to $\sim 50 \%$ of childhood disabilities in the United States $[1,2]$. The highest risks of morbidity and mortality are seen with the most premature infants [1]. Extremely premature ( $<27$ weeks' gestation), extremely low birth weight $(<1000 \mathrm{~g})($ EP-ELBW) infants' mortality rates are $30-50 \%$ [1]. Survivors face substantial risk of morbidities including intraventricular hemorrhage (IVH), chronic lung disease (CLD), and retinopathy of prematurity (ROP) [1]. These morbidities are predictors of long-term neurodevelopmental, sensorial, and psychiatric disabilities, which affect quality of life and can negatively impact the family [1,3-5].

Following birth, EP-ELBW infants are susceptible to rapid development of hypothermia, hypoglycemia, hypotension,

Sarah E. W. Croop

croop@email.unc.edu

1 Division of Neonatology, Department of Pediatrics, School of Medicine, University of North Carolina at Chapel Hill, Chapel Hill, NC, USA

2 School of Nursing, University of North Carolina at Chapel Hill, Chapel Hill, NC, USA and respiratory failure [1, 6-24]. Resuscitation in the delivery room (DR) and stabilization during admission to the neonatal intensive care unit (NICU) involves a series of interdependent tasks and procedures. These interventions must be performed quickly, proficiently, and systematically to minimize the short-term sequelae of prematurity, which contribute to the risk of long-term morbidity and mortality [1, 6-18].

The hour immediately following the birth of an EP-ELBW infant is referred to as the "Golden Hour" (GH) [6-8, 10-18]. This term denotes the period of time in which medical care to prevent irreversible damage is most effective and represents the inverse relationship between elapsing minutes and likelihood of survival. Due to the complexity of the care required at birth, protocols that bundle evidence-based practices and standardize their application to the first few minutes after birth have been used to improve the quality and consistency of care for EP-ELBW infants during the GH [6-8, 10-18].

\section{Problem}

In 2012, Vermont Oxford Network database reports for the NICU at the University of North Carolina (UNC) Children's Hospital revealed admission temperatures for EP-ELBW infants fell below the unit's goal range $\left(36.5-37.5^{\circ} \mathrm{C}\right)$, and the network's reported ranges for similar type NICUs. In our unit, $25 \%$ of infants $<24$ weeks' gestation and $41 \%$ of infants 
24-26 weeks' gestation were euthermic compared to 34 and $46 \%$ of similar type NICUs in Vermont Oxford Network.

A propensity for early hypoglycemia (glucose $<45 \mathrm{mg} /$ dL) was also noted. At baseline, $18 \%$ of EP-ELBW infants were hypoglycemic on admission. In addition, retrospective chart review demonstrated the time from birth to obtaining intravascular (IV) access, subsequent administration of IV fluids and antibiotics, and overall time to completion of admission stabilization was prolonged, increasing the risk for hypothermia and hypoglycemia.

\section{Available knowledge}

In a review of the literature, nine quality improvement (QI) studies were identified presenting outcomes following implementation of a GH protocol in the NICU [8, 10, 12-18]. Admission temperature was reported as a short-term outcome measure and improvements in temperatures to goal range were described in eight of the studies [10, 12-18]. One study provided statistical analysis of improvement in admission serum glucose concentration to $>50 \mathrm{mg} / \mathrm{dL}$ following protocol implementation [13]. Another described "stable" glucoses throughout the QI project [14]. The only other study that discussed glycemic control reported an increase in the rate of hypoglycemia which was attributed to increased physiologic stress [18]. Although long-term outcomes were not consistently evaluated in the studies, several authors described improvements in the incidence of IVH, ROP, CLD, late-onset sepsis, and/or length of stay in their units $[8,10,12,16,18]$.

\section{Specific aims}

Our goal was to design and implement a systematic, evidencebased approach to the care of EP-ELBW infants during the GH. The primary aims were to increase the percentage of temperatures within euthermic range, reduce the incidence of hypoglycemia, and decrease the time to completion of admission stabilization to less than 60 min after birth.

\section{Methods}

\section{Design}

A retrospective-prospective study design was used for comparison of data from a preprotocol cohort with postprotocol cohorts. Retrospective data were collected January, 2012 through March, 2014 from electronic medical records. Prospective data collection began with project implementation in April, 2014 and continued through February, 2015 for Phase I and March, 2015 through March, 2017 for Phase II (Table 1). The effectiveness of interventions was evaluated using plan- do-study-act (PDSA) cycles in accordance with institutional use of Lean Six Sigma QI methodology [25, 26].

\section{Setting and population}

This single-center, QI study was conducted in the 58-bed level IV NICU at UNC Children's Hospital, in Chapel Hill, NC. The NICU is part of a large academic medical center and serves as a regional referral center for the state. Nearly two-thirds of the 800-1000 infants admitted to the unit each year are inborn. The study population included all inborn infants $<27$ weeks' gestation who survived resuscitation in the DR and were admitted to the NICU. Exclusion criteria were major congenital anomalies resulting in death within the first $24 \mathrm{~h}$ after birth and/or parental decision to provide palliative care on admission.

\section{Context}

A multidisciplinary team of NICU providers including neonatologists, neonatal nurse practitioners, neonatal fellows, nurses, and respiratory therapists designed the evidence-based bundles included in the protocol. Representatives from each of these groups of key stakeholders were chosen to lead the proposed culture change. Prior to implementation, presentations demonstrating baseline variation in practices were given to increase awareness of the need for improvement. Disciplinary-specific education sessions were provided with each phase. To promote sustainability, GH care was incorporated into the nurses' yearly competencies and new fellows' bootcamp. Updates were provided throughout the project to maintain stakeholder engagement.

\section{Interventions}

\section{Thermoregulation}

To minimize heat loss, the DR temperature was increased $\left(74-76^{\circ} \mathrm{F}\right.$ ) prior to delivery and the radiant warmer was prepared in collaboration with the obstetrical team. In the NICU, the isolette and all supplies were warmed. Radiant heat, plastic wrap, and stocking-knit caps were used at delivery in the baseline cohort. With project implementation, a warming mattress was added and the technique for wrapping the infant in plastic was modified to improve integrity during resuscitation. One corner of the plastic was folded over the infant's head and secured with a stocking-knit cap, as plastic head-coverings have been shown to be more effective at preventing heat loss than use of stocking-knit caps alone [27]. In Phase II, the 


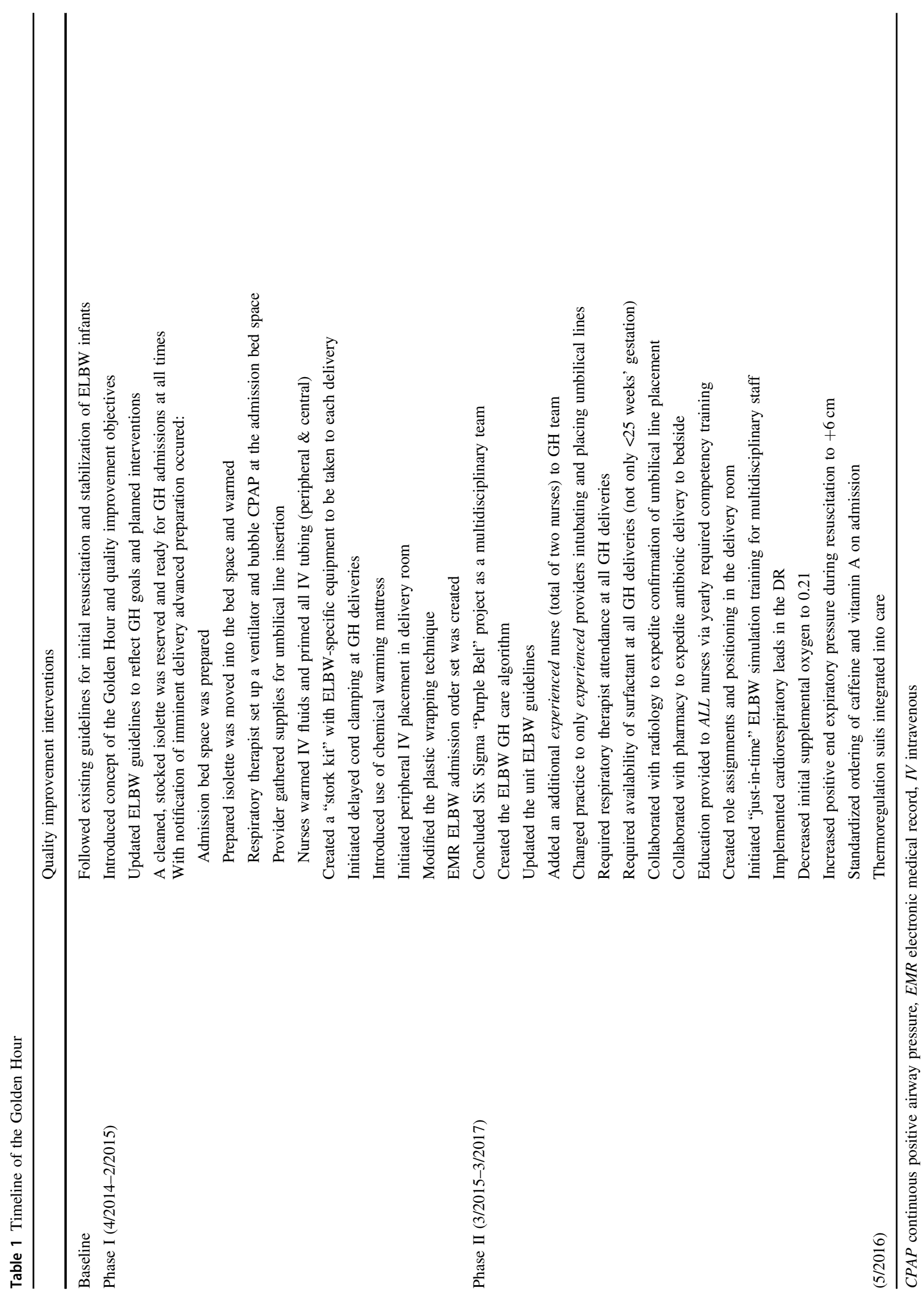


plastic wrap and stocking-knit cap were replaced by a polyethylene, heat loss prevention suit with a hood. The suit could be opened for central umbilical line placement without full removal, thus avoiding loss of heat and moisture during the procedure.

\section{Glycemic control}

To achieve early initiation of glucose infusion, peripheral IV access was established in the DR with a maximum of two attempts at placement. On admission to the NICU, a $10 \%$ dextrose infusion was administered peripherally while central umbilical access was being established. Following radiographic confirmation of placement, the peripheral infusion was discontinued, and central infusions were initiated using $10 \%$ dextrose, $2.5 \%$ amino acid solution via the umbilical venous catheter and $3.6 \%$ isotonic amino acid solution via the umbilical arterial catheter. A NICU-specific system for ordering umbilical line placement films was developed in collaboration with the radiology department to reduce time to confirmation of central placement.

\section{Infection control}

Establishing vascular access was also vital to treating potential sepsis. When indicated, antibiotics were started on admission to the NICU. All lab work including the blood culture was collected as soon as blood return was obtained during central umbilical line placement. Once the blood culture was collected, antibiotics could be administered peripherally before central line placement had been completed or confirmed. Collaboration with the pediatric pharmacy department improved timing of delivery of antibiotics to the bedside.

\section{Respiratory management}

A respiratory management plan for DR resuscitation was implemented, based on gestational age and risk factors for respiratory compromise (Fig. 1). This plan was used throughout the project in complement with the Neonatal Resuscitation Program (NRP) guidelines. Following publication of the 7th edition NRP guidelines, cardiorespiratory monitoring in the DR was implemented and initiation of supplemental oxygen was reduced from 0.3 to 0.21 (Table 1) [28]. Administration of intramuscular Vitamin A and intravenous caffeine was routine for the first two study cohorts. However, there were some inconsistencies with the timing of initiation. In an effort to improve outcomes, the initiation of these medications was standardized (Table 1) [29, 30]. During Phase II, both medications were added to the admission order set in the
EMR and ordered within the first hour after birth for all EPELBW infants.

\section{Teamwork and communication}

Many of the interventions implemented with Phase II of the project were focused on improving teamwork and communication. A detailed process flowchart was developed to guide the team through the GH (Fig. 1). At baseline, respiratory therapy presence at EP-ELBW deliveries was inconsistent. For implementation of Phase II, a respiratory therapist was assigned to attend every GH delivery. An additional, experienced nurse, and an additional, experienced provider were also added to the team. Previously, one provider assumed responsibility for establishing the airway and leading the team. With implementation of Phase II, two providers, physician or neonatal nurse practitioner, attended the delivery and divided these roles. The leader was responsible for supervising the resuscitation and stabilization of the infant from birth to completion of the GH as well as communicating with the obstetrical team and the family. The second provider focused on establishing an airway in the DR and inserting umbilical lines in the NICU. At this time, each team member was also assigned specific tasks and positions around the radiant warmer in the DR to improve team dynamics and communication [10].

Just-in-time training simulations were employed to solidify these interventions. When delivery of an infant $<27$ weeks' gestation was imminent, the team members assigned to attend reviewed their roles, responsibilities, and positions around the radiant warmer and performed a quick, just-in-time simulation. Consistent exposure to each team member's role created a shared mental model of what needed to be accomplished and who was responsible for each task throughout the GH. Consequently, the team was able to assume and divide additional responsibilities on the rare occasion one role was unfulfilled.

\section{Study of the interventions}

Initially, a basic handout reminded providers of the goals of the GH and served as the data collection tool. Subsequently, a detailed process flowchart with corresponding questions for data collection replaced the initial handout (Fig. 1). All information gathered from the data collection tools was confirmed in the medical record prior to analysis.

Each phase of the project corresponded to changes in the bundles of interventions (Table 1). Changes in interventions were determined using PDSA cycles, taking into consideration trends in outcomes, stakeholder feedback, and availability of new products and evidence-based practices. Evaluation of short-term outcomes and other process 


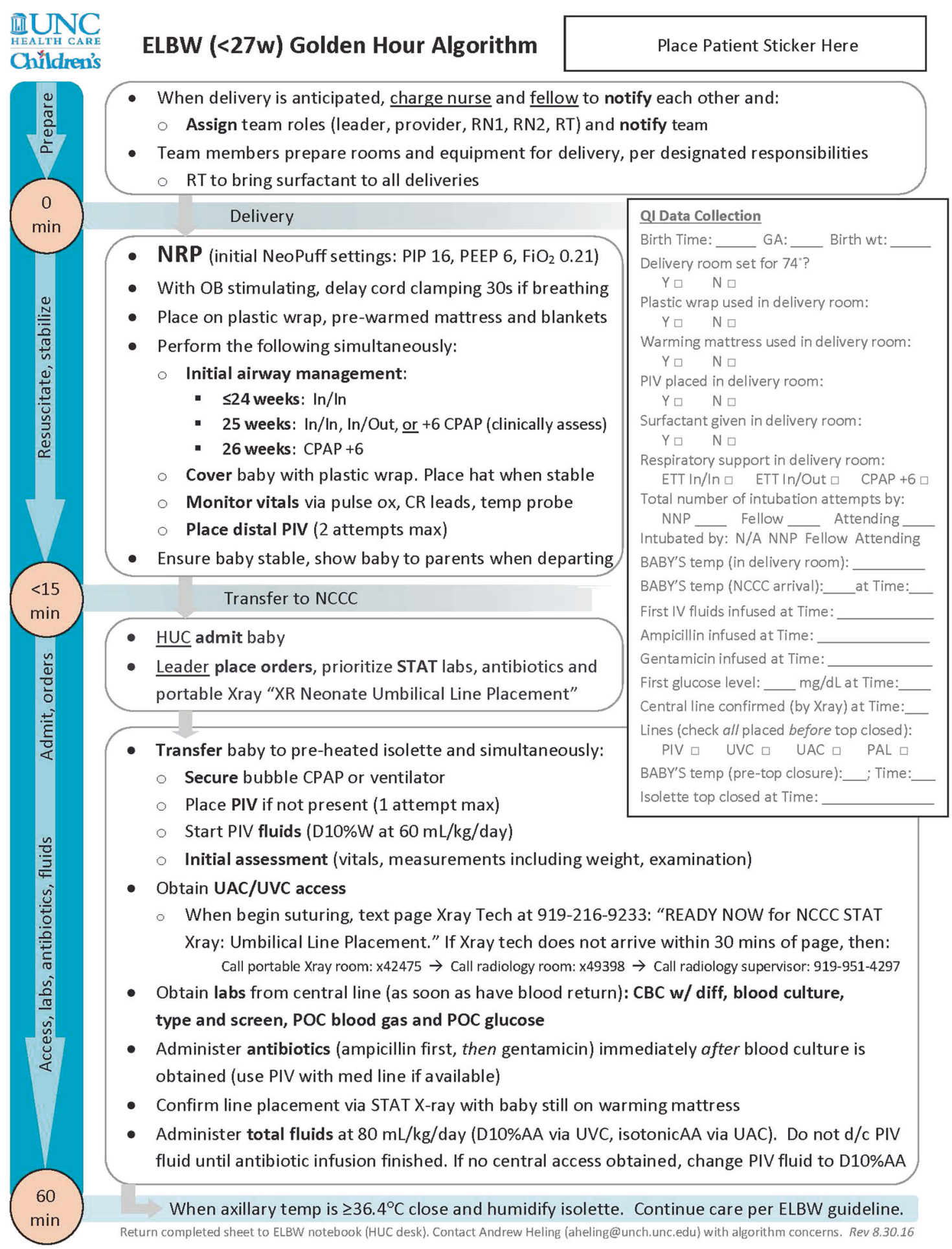

Fig. 1 Golden Hour algorithm initial airway management was based on the infant's gestational age and assessment of risk factors for respiratory failure. Choices included intubation with surfactant administration (In/In), intubation for surfactant administration with immediate extubation to CPAP (In/Out), or CPAP without surfactant administration. AA amino acid, CBC w/diff complete blood count with differential, CPAP continuous positive airway pressure, CR cardiorespiratory, D10\%W dextrose $10 \%$ in water; ETT endotracheal tube, $\mathrm{FiO}_{2}$ fraction of inspired oxygen, $\mathrm{HUC}$ hospital unit coordinator; NCCC Newborn Critical Care Center, NNP neonatal nurse practitioner, OB obstetrician, PAL peripheral arterial line, PEEP positive end expiratory pressure, PIP positive inspiratory pressure, PIV peripheral intravenous line, POC point-of-care, RN registered nurse, RT respiratory therapist, STAT immediately, UAC/UVC umbilical arterial and venous catheters 
measures including time to achieving various GH goals provided benchmarks for determining the effectiveness of interventions. To ensure internal validity, implementation fidelity was evaluated based on the consistency of adherence to protocol interventions.

\section{Measures}

Primary, short-term outcome measures were those obtained during admission stabilization. Axillary temperatures were measured on admission to the NICU (goal range 36.5-37.5 $\left.{ }^{\circ} \mathrm{C}\right)$. Serum glucose concentration was obtained during central line placement and measured using point-of-care testing (goal $\geq 45 \mathrm{mg} / \mathrm{dL}$ ). Completion of admission stabilization included DR resuscitation, initiation of IV fluids and antibiotics (when indicated), establishing central access, closing the top of the isolette, initiating humidity, and providing decreased environmental stimulation to approximate the intrauterine environment.

Process measures that directly contributed to thermoregulation, glycemic control, and time to completion of $\mathrm{GH}$ stabilization were tracked. These measures included the percentage of time plastic wrap or thermoregulation suit, warming mattresses, and peripheral IV insertion were utilized in the DR and minutes from birth to admission temperature, initiation of IV fluids, initiation of antibiotics, central line confirmation, and closure of the isolette.

Long-term outcomes including severe IVH (Grade III or IV hemorrhages), severe CLD defined as the need for supplemental oxygen $\geq 30 \%$ or positive pressure at 36 weeks' post menstrual age (PMA) or discharge [31], ROP requiring treatment, PMA on the day of discharge home, and mortality served as balancing measures to ensure changes in interventions were not having unintended, negative impacts.

\section{Analysis}

Statistical process control charts were created in QI Macros for Excel. Outcomes were compared with Chi-Square or Fisher's exact test for categorical data and one-way ANOVA or Kruskal-Wallis for continuous data using Stata statistical software. Median values with interquartile ranges are presented due to non-normal distribution of data. Statistical significance was set at $p$ values of $\leq 0.05$.

\section{Results}

The study included 214 inborn infants $<27$ weeks' gestation. Three infants were excluded due to severe congenital anomalies resulting in death shortly after admission. Data were collected over three phases; pre-protocol $(n=80)$, Phase I $(n=42)$, and Phase II $(n=92)$ (Table 1$)$. There were no significant differences in infant characteristics (Table 2).

Adherence to the intended interventions were as follows: the use of plastic wrap or thermoregulation suit was $100 \%$ and $98 \%$ in Phases I and II respectively and the use of warming mattresses was $95 \%$ in both phases. The number of attempts for IV insertion in the DR was limited (max of two) and overall, an IV was placed in the DR for $33 \%$ of infants in Phase I and $47 \%$ in Phase II.

Significant improvements were observed in all three primary outcome measures including the incidence of temperatures outside goal range $(59 \%$ vs $26 \%$ vs $38 \%$; $p=$ 0.001 ), the incidence of hypoglycemia ( $18 \%$ vs $7 \%$ vs $4 \%$; $p=0.012$ ), and median time in minutes to $\mathrm{GH}$ stabilization (110 vs 111 vs $92 \mathrm{~min} ; p=0.004$ ) (Table 3). Evaluation of long-term morbidity and mortality as balancing measures to stabilize our metrics did not reveal any unintended negative effects (Table 3).

Statistical process control charts were used to analyze process performance. Changes in the center line and control limits were based on process shifts that occurred with continuous evaluation and implementation of interventions throughout the project. At baseline, both processes were stable. A few instances of a single measure above the upper control limit or below the lower control limit were noted on both control charts. These special cause variations were related to deviation from or noncompliance with the process. New control limits were calculated for both charts when clinically relevant special cause variation was identified (Fig. 2a, b). The limits were recalculated for admission temperature two months after project implementation resulting in a new center line with a euthermic temperature $36.8^{\circ} \mathrm{C}$ (Fig. 2a). The limits were recalculated for time to completion during Phase II resulting in a new center line at $89 \mathrm{~min}$ (Fig. 2b). Both processes remained stable around the new center line.

\section{Discussion}

\section{Summary}

Implementation of an evidence-based $\mathrm{GH}$ protocol was effective at significantly improving euglycemia, euthermia, and time to completion of admission stabilization for EPELBW infants in our NICU. Following project implementation, admission temperatures in our unit for infants $<27$ weeks' gestation consistently fell within the Vermont Oxford Network's reported ranges for similar type NICUs. Although we did not consistently meet our goal of completion within the first $60 \mathrm{~min}$ after birth, we made significant improvements in time from baseline. 
Table 2 Golden Hour patient characteristics

Table 3 Golden hour outcomes

\begin{tabular}{llcccc}
\hline Variable & Statistic & $\begin{array}{l}\text { Preprotocol } \\
(N=80)\end{array}$ & $\begin{array}{l}\text { Phase I } \\
(N=42)\end{array}$ & $\begin{array}{l}\text { Phase II } \\
(N=92)\end{array}$ & $P$ \\
\hline Gestational age (weeks) & Median (IQR) & $25(24,26)$ & $25(24,26)$ & $25(23,26)$ & 0.44 \\
Birth weight (g) & Median (IQR) & $705(607,854)$ & $740(650,860)$ & $670(580,805)$ & 0.13 \\
Female sex & $N(\%)$ & $38(48)$ & $22(52)$ & $50(54)$ & 0.66 \\
Antenatal steroids & $N(\%)$ & $74(93)$ & $42(100)$ & $88(96)$ & 0.18 \\
Cesarean delivery & $N(\%)$ & $53(66)$ & $30(71)$ & $57(62)$ & 0.56 \\
5-min Apgar & Median (IQR) & $5(3,7)$ & $6(3,8)$ & $6(3,7)$ & 0.56 \\
Surfactant at delivery & $N(\%)$ & $47(59)$ & $22(52)$ & $51(55)$ & 0.79 \\
Surfactant by 2 h & $N(\%)$ & $59(74)$ & $28(67)$ & $72(78)$ & 0.37 \\
\hline
\end{tabular}

$I Q R$ interquartile range

\begin{tabular}{lllcll}
\hline Variable & Statistic & $\begin{array}{l}\text { Preprotocol } \\
(N=80)\end{array}$ & $\begin{array}{l}\text { Phase I } \\
(N=42)\end{array}$ & $\begin{array}{l}\text { Phase II } \\
(N=92)\end{array}$ & $P$ \\
\hline Severe IVH & $N(\%)$ & $16(20)$ & $5(14)$ & $16(18)$ & 0.722 \\
Survivors without Severe CLD & $N(\%)$ & $38(58)$ & $19(66)$ & $28(45)$ & 0.140 \\
Survivors without ROP treatment & $N(\%)$ & $56(84)$ & $27(90)$ & $47(72)$ & 0.100 \\
Death & $N(\%)$ & $12(15)$ & $11(26)$ & $23(25)$ & 0.186 \\
Death or CLD & $N(\%)$ & $39(51)$ & $21(53)$ & $57(67)$ & 0.077 \\
PMA at discharge home (weeks) & Median (IQR) & $40(38,43)$ & $41(38,44)$ & $41(39,45)$ & 0.240 \\
Time to completion (minutes) & Median (IQR) & $110(89,138)$ & $111(94,135)$ & $92(74,129)$ & 0.004 \\
Non-euthermic temperature & $N(\%)$ & $46(59)$ & $11(26)$ & $33(38)$ & 0.001 \\
Hypoglycemic & $N(\%)$ & $14(18)$ & $3(7)$ & $4(4)$ & 0.012 \\
\hline
\end{tabular}

Euthermic range $\left(36.5-37.5^{\circ} \mathrm{C}\right)$. Hypoglycemia defined as glucose $<45 \mathrm{mg} / \mathrm{dL}$

$C L D$ chronic lung disease, $I Q R$ interquartile range, $I V H$ intraventricular hemorrhage, $P M A$ postmenstrual age, $R O P$ retinopathy of prematurity

\section{Interpretation}

Our GH protocol was implemented in a more premature population of infants than those of the studies in our review. Only one of the review studies focused on a population of infants $<27$ weeks' gestation, while inclusion criteria for the remaining studies ranged from $<28$ to $<33$ weeks' gestation $[8,10,12-18]$. However, despite a more vulnerable population, the improvements in admission glucoses and temperatures demonstrated in our study were consistent with the results reported by the studies in our review. Initially, we noted a slight increase in time which we contributed to the learning curve associated with project implementation. The significant decrease in time to GH stabilization during Phase II was associated with efforts to improve team dynamics and implementation of simulation training.

A potential confounder was the increase in the resuscitation and survival of periviable infants (22-23 weeks' gestation) during the course of our interventions, a phenomenon which was not isolated to our unit $[32,33]$. We noted that the percent of periviable infants remained stable across the first two cohorts and nearly doubled in the third cohort $(16 \%, 16 \%$, and $29 \%$ respectively). The inverse relationship between gestational age and survival without morbidities is well-recognized and confers a higher degree of vulnerability to those infants delivered at the "cusp of viability" [1,34]. Periviable infants are more susceptible to hypothermia and hypoglycemia and less resilient from the ensuing morbidities [35]. Despite an increasing percentage of more immature infants, the rates of hypothermia and hypoglycemia improved without significant differences in balancing measures. This finding remained when balancing measures were evaluated separately for 22 and 23 week infants. We speculate that implementation of the interventions in our GH protocol may offer a protective effect which may be even more beneficial at lower gestational ages.

\section{Limitations}

One limitation to the study was the transfer of stable infants to lower-level facilities. There was a loss of $7 \%$ of the data for CLD and $4 \%$ of the data related to ROP for survivors transferred prior to 36 weeks' PMA. Sample size was also a limiting factor. The sample size for Phase I of the project 
a

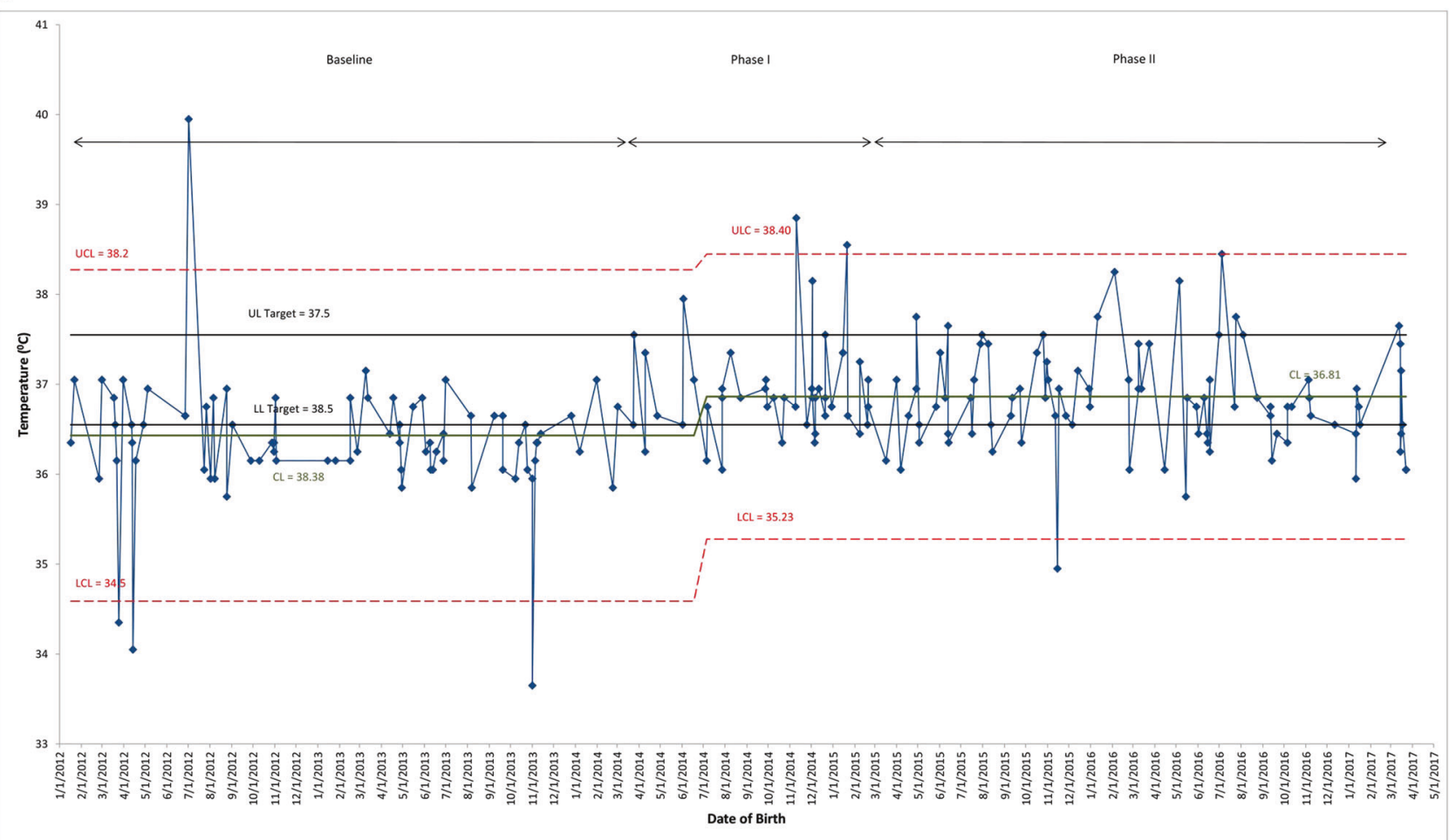

b

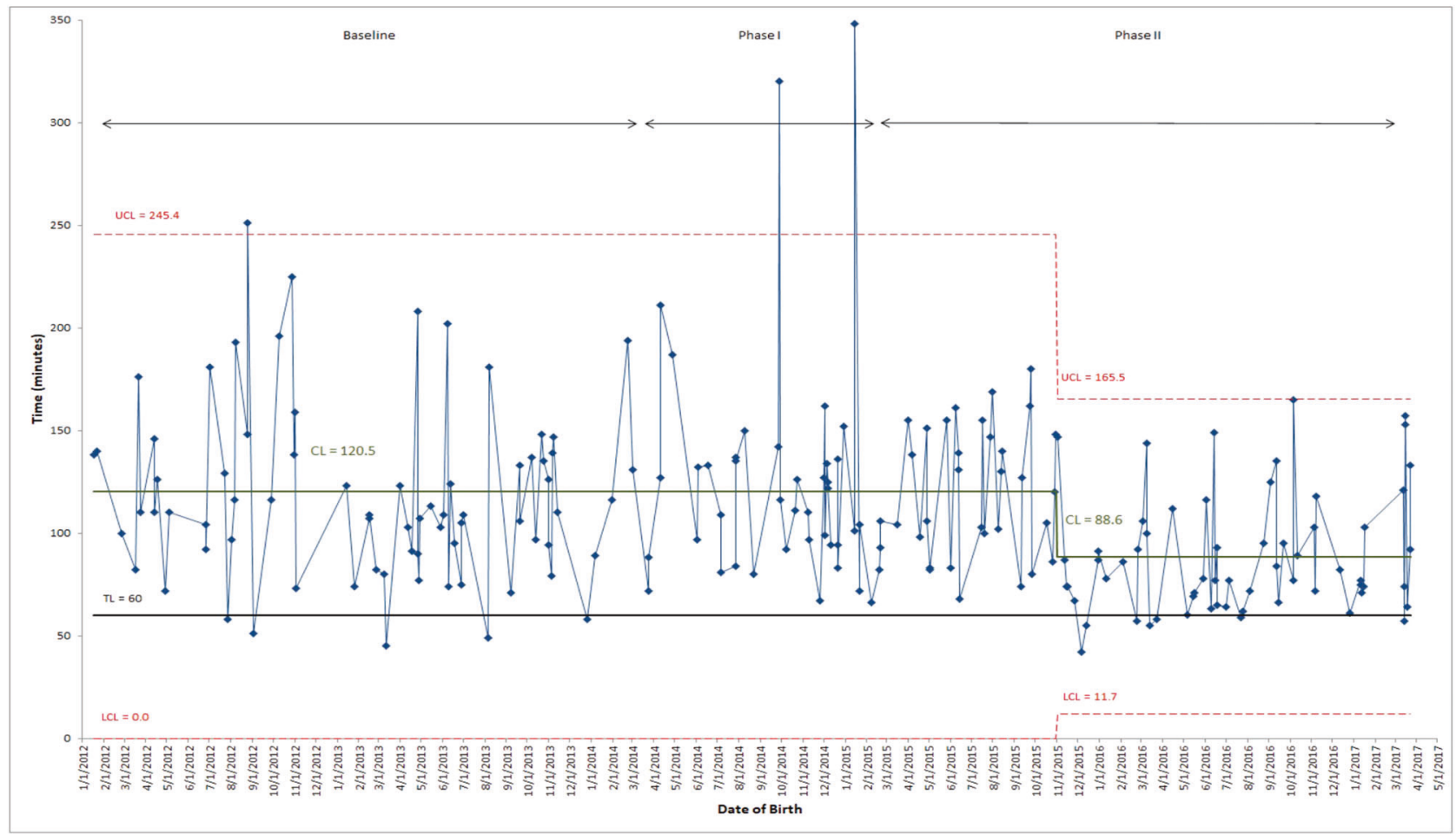

Fig. 2 Golden Hour statistical process control charts a Change in admission temperatures over time b Change in time to completion of admission stabilization over time. LCL lower control limit, UCL upper control limit 
was approximately $50 \%$ that of the baseline and Phase II cohorts. Although no power analysis was performed, it is likely that the sample size of this study was not adequate to assess for differences in long-term outcomes which were used as balancing measures.

The 5-year span of this project and the retrospective/ prospective cohort design introduce the risk of historical threat to the long-term outcomes which served as balancing measures. Although we saw no significant differences in the rates of mortality, IVH, CLD, or ROP we recognize that these disease processes are multifactorial in etiology. Changes in practice over time and other confounding variables, not related to the interventions of the GH might contribute to variations in outcomes and in some instances, provide an alternative explanation of the study findings. Other NICUs may not have been exposed to the same changes in practice or confounding factors thus limiting the generalizability of the study. The study was a single-center QI project and the generalizability of the protocol is also limited as a result of potential differences in resources, staffing, and physical space. However, transferability is comparatively high as the evidencebased practice bundles that make up the protocol can be modified and adapted to fit the needs of any NICU providing care to EP-ELBW infants.

\section{Conclusion}

GH protocols prioritize maintaining the stability of the most vulnerable population of infants in the NICU through the first, tenuous minutes after birth. These protocols promote efficient care delivery and provide a structure for effective teamwork and communication across multidisciplinary groups. The findings of our study and their consistency with the findings of previous studies support implementation of GH protocols in the NICU to decrease admission hypothermia and hypoglycemia in EP-ELBW infants. Our findings also fill a gap in the literature related to the care of the periviable infant, suggesting GH protocols may be even more beneficial in this subpopulation.

Acknowledgements The authors would like to thank several team members who contributed significantly to the project's success: Andrew Heling, MD, Sue Meier, ND, NNP-BC, Tina DeLoache, MSN, NNP-BC, Anne Poisson, RN, Shannon Heath, RN, Vicki Childers, RN, and the entire staff of the Newborn Critical Care Center at the University of North Carolina at Chapel Hill Children's Hospital for their dedication to the care of our EP-ELBW infants. Vermont Oxford Network unpublished data used with permission.

\section{Compliance with ethical standards}

Conflict of interest The authors declare that they have no conflict of interest.
Ehtical approval In accordance with ethics and human subject permissions, this study was submitted to the Institutional Review Board of UNC, Chapel Hill and deemed exempt.

Publisher's note Springer Nature remains neutral with regard to jurisdictional claims in published maps and institutional affiliations.

\section{References}

1. Glass HC, Costarino AT, Stayer SA, Brett C, Cladis F, Davis PJ. Outcomes for extremely premature infants. Anesth Analg. 2015;120:1337-51. https://doi.org/10.1213/ANE. 0000000000000705 .

2. U.S. Department of Health and Human Services, Health Resources and Services Administration, Maternal and Child Health Bureau. Child Health USA 2014. Rockville, MD: U.S. Department of Health and Human Services; 2015. http://mchb.hrsa.gov/ chusa14.

3. Leversen KT, Sommerfelt K, Rønnestad A, et al. Prediction of neurodevelopmental and sensory outcome at 5 years in Norwegian children born extremely premature. Pediatrics. 2011;127: e630-e638. https://doi.org/10.1542/peds.2010-1001.

4. Fevang, SKE, Hysing, M, Markestad, T, Sommerfelt, K. Mental health in children born extremely preterm without severe neurodevelopmental disabilities. Pediatrics. 2016;137. https://doi.org/ 10.1542/peds.2015-3002.

5. Behrman, RE, Butler, AS, editors. Preterm birth: causes, consequences, and prevention. Washington, DC: National Academies Press; 2007. https://doi.org/10.17226/11622.

6. Vento M, Cheung P, Aguar M. The first golden minutes of the extremely-low-gestational-age neonate: a gentle approach. Neonatology. 2009;95:286-98. https://doi.org/10.1159/000178770.

7. Annibale DJ, Bissinger RL. The golden hour. Adv Neonatal Care. 2010;10:221-3. https://doi.org/10.1097/ANC.0b013e3181e9e244.

8. Reuter S, Messier S, Steven D. The neonatal golden hourintervention to improve quality of care of the extremely low birth weight infant. S D J Med. 2014;67:397-405.

9. Taylor SN, Kiger J, Finch C, Bizal D. Fluid, electrolytes, and nutrition: minutes matter. Adv Neonatal Care. 2010;10:248-55. https://doi.org/10.1097/ANC.0b013e3181f0823e.

10. Vergales BD, Dwyer EJ, Wilson SM, Nicholson EA, Nauman RC, Jin L, et al. NASCAR pit-stop model improves delivery room and admission efficiency and outcomes for infants $<27$ weeks' gestation. Resuscitation. 2015;92:7-13. https://doi.org/10.1016/j. resuscitation.2015.03.022.

11. Doyle KJ, Bradshaw WT. Sixty golden minutes. Neonatal Netw. 2012;31:289-94. https://doi.org/10.1891/0730-0832.31.5.289.

12. Ashmeade TL, Haubner L, Collins S, Miladinovic B, Fugate K. Outcomes of a neonatal golden hour implementation project. Am J Med Qual. 2014;31:73-80. https://doi.org/10.1177/ 1062860614548888.

13. Castrodale V, Rinehart S. The golden hour: improving the stabilization of the very low birth-weight infant. Adv Neonatal Care. 2014;14:9-14. https://doi.org/10.1097/ANC.0b013e31828d0289.

14. Lambeth TM, Rojas MA, Holmes AP, Dail RB. First golden hour of life: a quality improvement initiative. Adv Neonatal Care. 2016;6:264-72. https://doi.org/10.1097/ANC.0000000000000306.

15. Reynolds RD, Pilcher J, Ring A, Johnson R, McKinley P. The Golden Hour: care of the LBW infant during the first hour of life one unit's experience. Neonatal Netw. 2009;28:211-9. BP7L4623W07807L7.

16. Wallingford B, Rubarth L, Abbott A, Miers LJ. Implementation and evaluation of "golden hour" practices in infants younger than 
33 weeks' gestation. Newborn Infant Nurs Rev. 2012;12:86-96. https://doi.org/10.1053/j.nainr.2012.03.008.

17. Harriman TL, Carter B, Dail RB, Stowell KE, Zukowsky K, Harris-Haman PA. Golden hour protocol for preterm infants. Adv Neonatal Care. 2018;18:462-70. https://doi.org/10.1097/ANC. 0000000000000554 .

18. Peleg B, Globus O, Granot M, Leibovitch L, Mazkereth R, Eisen I. et al. "Golden Hour" quality improvement intervention and shortterm outcome among preterm infants. J Perinatol. 2019;39:387-92. https://doi.org/10.1038/s41372-018-0254-0.

19. McGrath JM. Is evidence-based practice routine in the golden hour? J Perinat Neonatal Nurs. 2012;26:109-11. https://doi.org/ 10.1097/JPN.0b013e318253e1f5.

20. Baumgart S. Iatrogenic hyperthermia and hypothermia in the neonate. Clin Perinatol. 2008;35:183-97. https://doi.org/10.1016/ j.clp.2007.11.002.

21. Bissinger RL, Annibale DJ. Thermoregulation in very low-birthweight infants during the golden hour: results and implications. Adv Neonatal Care. 2010;10:230-8. https://doi.org/10.1097/ANC. 0b013e3181f0ae63.

22. Laptook AR, Watkinson M. Temperature management in the delivery room. Semin Fetal Neonatal Med. 2008;13:383-39. https://doi.org/10.1016/j.siny.2008.04.003.

23. Miller SS, Lee HC, Gould JB. Hypothermia in very low birth weight infants: distribution, risk factors and outcomes. J Perinatol. 2011;31:49-56. https://doi.org/10.1038/jp.2010.177.

24. Laptook AR, Salhab W, Bhaskar B.The Neonatal Research Network. Admission temperature of low birth weight infants: predictors and associated morbidities. Pediatrics. 2007;119: e643-9.

25. QI training and education: Lean Six Sigma. Institute for Healthcare Quality Improvement. UNC School of Medicine website. https://www.med.unc.edu/ihqi/training/lean-six-sigma/. 2015. Accessed 13 Jan 2019.
26. Schweikhart SA, Dembe AE. The applicability of Lean and Six Sigma techniques to clinical and translational research. J Investig Med. 2009;57:748-55. 10.231/JIM.0b013e3181b91b3a.

27. Trevisanuto D, Doglioni N, Cavallin F, Parotto M, Micaglio M, Zanardo V. Heat loss prevention in very preterm infants in delivery rooms: a prospective, randomized, controlled trial of polyethylene caps. J Pediatr. 2010;156:914-7.

28. Weiner GM, Zaichkin J, Kattwinkel J, editors. Textbook of neonatal resuscitation. 7th ed. Elk Grove Village, IL: American Academy of Pediatrics and American Heart Association; 2016.

29. Darlow BA, Graham PJ, Rojas-Reyes MX. Vitamin A supplementation to prevent mortality and short- and long-term morbidity in very low birth weight infants. Cochrane Database Syst Rev. 2016;8: CD000501 https://doi.org/10.1002/14651858.CD000501.pub4.

30. Schmidt B, Roberts R, Davis P, et al. Caffeine therapy for apnea of prematurity. N Engl J Med. 2006;354:2112-21. https://doi.org/ 10.1056/NEJMoa054065.

31. Jobe AH, Bancalari E. NICHD/NHLBI/ORD workshop summary: bronchopulmonary dysplasia. Am J Respir Crit Care Med. 2001;163:1723-9.

32. Mahgoub L, van Manen M, Byrne P, Tyebkhan JM. Policy change for infants born at the "cusp of viability": a Canadian NICU experience. Pediatrics. 2014;134:e1405-10.

33. Mehler K, Oberthuer A, Keller T, et al. Survival among infants born at 22 or 23 weeks' gestation following active prenatal and postnatal care. JAMA Pediatr. 2016;170:671-7. https://doi.org/10. 1001/jamapediatrics.2016.0207.

34. Stoll BJ, Hansen NI, Bell EF, et al. Neonatal outcomes of extremely preterm infants from the NICHD Neonatal Research Network. Pediatrics.2010;126:443-56. https://doi.org/10.1542/peds. 2009-2959.

35. Wyckoff MH. Initial resuscitation and stabilization of the periviable neonate: the golden-hour approach. Semin Perinatol. 2014;38:12-16. https://doi.org/10.1053/j.semperi.2013.07.003. 